\title{
A CONSTRUCTION OF MAXIMALLY RECOVERABLE CODES
}

\author{
ALEXANDER BARG, ZITAN CHEN, AND ITZHAK TAMO
}

\begin{abstract}
We construct a family of linear maximally recoverable codes with locality $r$ and dimension $r+1$. For codes of length $n$ with $r \approx n^{\alpha}, 0 \leq \alpha \leq 1$ the code alphabet is of the order $n^{1+3 \alpha}$, which improves upon the previously known constructions of maximally recoverable codes.
\end{abstract}

\section{INTRODUCTION}

Consider a linear code $\mathcal{C}$ over a finite field $F=\mathbb{F}_{q}$ of length $n$ and dimension $k$, and let $r$ be a number such that $r+1$ divides $n$. We will write $[n]=\left\{(i, j), j=1, \ldots, r+1 ; i=1, \ldots, \frac{n}{r+1}\right\}$, and for $i=1, \ldots, \frac{n}{r+1}$ we will call the subset of indices $R_{i}=\{(i, j), j=1, \ldots, r+1\}$ a repair group. Call a set $T \subset[n]$ a transversal of the set of repair groups $\mathscr{R}=\left(R_{i}\right)_{i}$ if $\left|T \cap R_{i}\right|=1$ for all $i$. For a subset $X \subset[n]$ denote by $\left.\mathcal{C}\right|_{X}$ the puncturing of $\mathcal{C}$ in the coordinates in $X$, i.e., a coordinate projection of $\mathcal{C}$ on the complement of $X$ in $[n]$.

Definition 1. The code $\mathcal{C}$ is called maximally recoverable $(M R)$ with locality $r$ if the following two properties hold:

(i) For any repair group $R_{i}$ the code $\left.\mathcal{C}\right|_{R_{i}^{c}}$ has distance at least 2 ;

(ii) For any transversal $T$ of $\mathscr{R}$ the code $\left.\mathcal{C}\right|_{T}$ is maximum distance separable.

We write the parameters of the code $\mathcal{C}$ as $(n, k, r)=$ (length,dimension,locality).

This definition is a particular case of a more general notion of MR codes introduced in [5]. Namely, one assumes that (i), any repair group is capable of correcting any $a \geq 1$ erasures, and (ii), upon puncturing any $a$ coordinates from each of the repair groups, the punctured code is maximum distance separable that can correct $h$ erasures. Thus, Definition 1 corresponds to the case of $a=1$ and $h=\frac{n r}{r+1}-k$.

The main problems related to MR codes are: the minimum field size $q$ required to construct an MR code with a given set of parameters, and finding explicit constructions of MR codes, with sizeable literature devoted to them over the last decade. In this note we prove the following result.

Theorem 1. There exists a family of $(n, k=r+1, r)$ MR codes over $\mathbb{F}_{q}$, where $r=\Theta\left(n^{\alpha}\right), 0 \leq \alpha \leq 1$, with $q=\Theta\left(n^{1+3 \alpha+o(1)}\right)$.

To establish it, we develop an idea behind one construction in [6] that gave codes with locality $r=3$ and large $k$ relying on Behrend's classic result on sets of integers with no 3-term arithmetic progressions [2]. To address the case of general $r$ we use Alon's extension of Behrend's construction [1].

For the context we include a sample of the known results on the construction and parameters of MR codes, focusing on the regime of large $h$ relevant to us. Among the known families of MR codes we note constructions with $q=O\left((r+1) n^{2 h-1}\right)$ [4], $q=O\left((r+1)^{\frac{n r}{r+1}}\right)$ [9], $q=O\left(\max \left(r+1, \frac{n}{r+1}\right)\right)^{h}$ [3], as well as a number of constructions in [7] with comparable parameters. We refer the reader to the introduction of [6,7], or [3], Table 1, for a more detailed overview which also covers the entire range of possible parameters $a, r$, and $h$. As remarked in [7], most of the known constructions require alphabets of size $q$ that depend exponentially on $h$. One exception where the minimum alphabet size is independent of $h$ is the construction of [8] which requires $q=O(\max (n /(r+1), r+1))^{r}$. This is larger than the result in Theorem 1 above both for fixed and growing $r$. In summary, the code family constructed in this paper improves upon the known results in terms of the required field size.

To address the question of lower bounds (impossibility), we observe that the known nontrivial results [6] assume that $h$ is a fixed constant. At the same time, in our setting $h$ is clearly increasing with $n$, and the only 
known constraints on $q$ are general bounds of the form $q=\Omega(n)$ (for instance, part (ii) of Definition 1 implies that $q \geq k+1$, see [5, Theorem 19]).

\section{THE CONSTRUCTION}

Let $\gamma$ be a primitive element of $F$ and let $N=q-1$ be the size of its multiplicative group. We will define a linear code over $F$ by specifying its generator matrix $G=\left(g_{\ell,(i, j)}\right)$ of dimensions $(r+1) \times n$ where the length $n$ will be determined later. Let

$$
\mathcal{A}=\bigcup_{i=1}^{\frac{n}{r+1}} A_{i} \subset \mathbb{Z}_{N}
$$

be a subset of size $n$ formed as a union of pairwise disjoint sets $A_{i}$, and let $\left\{a_{i j}, j=1, \ldots, r+1\right\}$ be the elements of $A_{i}, i=1, \ldots, n /(r+1)$. Define

$$
g_{\ell,(i, j)}= \begin{cases}\gamma^{\ell \cdot a_{i j}} & 1 \leq \ell \leq r \\ \gamma^{(r+1) \cdot a_{i j}}+(-1)^{r+1} & \ell=r+1\end{cases}
$$

The main property of the set $\mathcal{A}$ that supports the construction is the following: let $\left\{a_{1}, \ldots, a_{r+1}\right\} \subset \mathcal{A}$, then

$$
\sum_{s=1}^{r+1} a_{s}=0(\bmod N) \text { if and only if } \exists i \in\left\{1, \ldots, \frac{n}{r+1}\right\} \text { s.t. } A_{i}=\left\{a_{1}, \ldots, a_{r+1}\right\} .
$$

Theorem 2. Let $G$ be the matrix defined above, where the set $\mathcal{A}$ satisfies (2). Then the rows of $G$ span an $(n, r+1, r)$-MR code over $F$.

Next we give a construction of the set $\mathcal{A}$ with the required properties. Let $\lambda$ and $\delta$ satisfy $0<\lambda<\frac{1}{r^{3}}, 0<$ $\delta<\frac{\lambda}{r}$, and define $d=\lfloor\delta N\rfloor, l=\lfloor\lambda N\rfloor$. Suppose that $D \subset\{1, \ldots, d\}$ is a subset of integers such that for any $d_{0}, \ldots, d_{r} \in D$ the equation over $\mathbb{Z}$

$$
d_{0}+\cdots+d_{r-1}=r d_{r}
$$

is satisfied only if $d_{0}=d_{1}=\cdots=d_{r}$. Define $r+1$ subsets $D_{i} \subset \mathbb{Z}_{N}$ by letting

$$
D_{i}= \begin{cases}i l+D & 0 \leq i \leq r-1 \\
N-\left(\begin{array}{l}
r \\
2
\end{array}\right) l-r \cdot D & i=r\end{cases}
$$

where $b+D, b \cdot D$ mean adding or multiplying every element of $D$ by $b$. By the choice of $\lambda$ and $\delta$ one can verify that the subsets $D_{i}$ are disjoint. Define the set $\mathcal{A}=\cup_{i=0}^{r} D_{i}$ and note that $|\mathcal{A}|=n:=|D|(r+1)$. Consider a partition of $\mathcal{A}$ into disjoint transversals $A_{b}$ for any $b \in D$ where

$$
A_{b}=\left\{a_{i, b}: i=0, \ldots, r\right\} \text { and } a_{i, b}= \begin{cases}i l+b, & 0 \leq i \leq r-1 \\
N-\left(\begin{array}{c}
r \\
2
\end{array}\right) l-r b, & i=r\end{cases}
$$

Lemma 3. The partition $\mathcal{A}=\cup_{b \in D} A_{b}$ satisfies property (2).

Large sets of integers that satisfy (3) exist, namely, the following is true.

Lemma 4. [1, Lemma 3.1] For every $r \geq 2$ and every positive integer $m$, there exists a subset $D \subset$ $\{1,2, \ldots, m\}$ of size at least

that has property (3).

$$
|D| \geq \frac{m}{e^{5 \sqrt{\log m \log r}}}
$$

This claim is proved by an averaging argument over intersections of a subset of integers with spheres of varying radii, and this is the only non-explicit part of our construction. We include a short proof at the end of the next section to make the presentation self-contained.

Putting things together, we have constructed an $(n, r+1, r)$ MR code $\mathcal{C}$ of length

$$
n=|D|(r+1) \geq(r+1) d e^{-5 \sqrt{\log d \log r}},
$$


where we have used Lemma4 with $m=d$. Let us estimate the dependence of the field size $q$ on the parameters of $\mathcal{C}$, letting $n, q \rightarrow \infty$. We have

$$
\log n \geq \Omega\left(\log q-3 \log r-5 \sqrt{\log \frac{q}{r^{4}} \log r}\right) .
$$

where we put $d=\Theta\left(\frac{q}{r^{4}}\right)$ (this appears to be the best choice given our assumption on $\delta$ ). Suppose that $r=$ $\Theta\left(n^{\alpha}\right)$, where $0 \leq \alpha \leq 1$ and note that this includes the cases of constant $r$ and various rates of increase of $r$ up to $r=\Theta(n)$, i.e., a constant number of repair groups. Now from (6) we obtain the estimate for $q$ stated in Theorem 1

\section{ProOfS}

Proof of Theorem 2 Let $S \subseteq[n]$ be an $(r+1)$-subset of indices and let $G_{S}$ be a square submatrix of $G$ of order $r+1$ whose columns are indexed by the elements of $S$. We begin by showing that the rank of $G_{S}$ is $r$ if $S$ forms a repair group, otherwise, $G_{S}$ is of full rank. First note that the first $r$ rows of $G_{S}$ form an $r \times(r+1)$ Vandermonde submatrix, hence the rank of $G_{S}$ is at least $r$. The rank is exactly $r$ if and only if there exists a nonzero vector $f=\left(f_{1}, \ldots, f_{r}, f_{r+1}\right)$ such that $f \cdot G_{S}=0$. Note that $f_{r+1} \neq 0$ since otherwise, it would violate the fact that the first $r$ rows of $G_{S}$ are linearly independent. Therefore, assume wlog that $f_{r+1}=1$. Since the columns of $G$ are defined by elements $\gamma^{\beta}, \beta \in \mathcal{A}$, the conditions $f \cdot G_{S}=0$ are alternatively written as $f\left(\gamma^{\beta_{i}}\right)=0$ for $i=1, \cdots, r+1$ and some $\beta_{i} \in \mathcal{A}$, where

$$
f(x):=x^{r+1}+(-1)^{r+1}+\sum_{i=1}^{r} f_{i} x^{i} .
$$

By assumption, the monic polynomial $f(x)$ has $r+1$ zeros $\gamma^{\beta_{i}}$, and thus

$$
f(x)=\prod_{i=1}^{r+1}\left(x-\gamma^{\beta_{i}}\right) .
$$

By comparing the constant terms in the two expressions of $f$ we have

$$
(-1)^{r+1}=\prod_{i=1}^{r+1}\left(-\gamma^{\beta_{i}}\right)=(-1)^{r+1} \gamma^{\sum_{i=1}^{r+1} \beta_{i}}
$$

or $\sum_{i=1}^{r+1} \beta_{i}=0(\bmod N)$. Then, by recalling assumption (2), either the subset $S$ forms a repair group, or otherwise $G_{S}$ is of full rank. Hence property (ii) in Definition 1 holds. Next, assume that $S$ forms a repair group, and we need to show that $\left.\mathcal{C}\right|_{S^{c}}$ has distance at least 2 . To prove this, note that any $(r+1) \times r$ submatrix of $G_{S}$ has rank $r$ since it contains an $r \times r$ Vandermonde submatrix. Since $\operatorname{rk}\left(G_{S}\right)=r$, any column of $G_{S}$ is in the span of the remaining $r$ columns, and thus the code $\left.\mathcal{C}\right|_{S^{c}}$ corrects a single erasure.

Proof of Lemma 3 . Let $\mathscr{B}:=\left\{b_{0}, b_{1}, \ldots, b_{r}\right\} \subset \mathcal{A}$. We will show that

$$
\sum_{i=0}^{r} b_{i}=0(\bmod N)
$$

is satisfied if and only if $\mathscr{B}$ coincides with one of the transversals $A_{b}$ defined in (5). One direction is easy: namely, the elements in every $A_{b}$ sum to 0 modulo $N$. Indeed

$$
\sum_{j=0}^{r} a_{i, b}=\sum_{j=0}^{r-1}(i l+b)+N-\left(\begin{array}{l}
r \\
2
\end{array}\right) l-r b=0(\bmod N) .
$$

Conversely, suppose (7) holds. We aim to prove that $\mathscr{B}=A_{b}$ for some $b \in D$. Let $t=\left|\mathscr{B} \cap D_{r}\right|$ and let us first show that $t=1$. Indeed, if $t=0$, then each $b_{i} \notin D_{r}$ and therefore $b_{i} \leq(r-1) l+d$ over $\mathbb{Z}$, and (again over $\mathbb{Z}$ )

$$
0<\sum_{i=0}^{r} b_{i} \leq r((r-1) l+d)<r^{2} l<N,
$$


where the last inequality follows by the choice of $\lambda$. This contradicts (7), so $t \geq 1$. A similar argument applies in the case of $t \geq 2$, namely we will show that in such a case $(t-1) N<\sum_{i=0}^{r} b_{i}<t N$ over $\mathbb{Z}$, which again will contradict (7). Indeed, we have

$$
\begin{aligned}
\sum_{i=0}^{r} b_{i} & >\sum_{b_{i} \in D_{r}} b_{i} \geq t N-t\left(\begin{array}{l}
r \\
2
\end{array}\right) l-t r d \geq(t-1) N+N\left(1-t \lambda\left(\left(\begin{array}{l}
r \\
2
\end{array}\right)+1\right)\right) \\
& =(t-1) N+N\left(1-t \lambda \frac{r^{2}-r+2}{2}\right)>(t-1) N,
\end{aligned}
$$

where the last step follows since $\lambda<r^{-3}, t \leq r+1$ and $r \geq 2$. For the upper bound write

$$
\begin{aligned}
\sum_{i=0}^{r} b_{i} & =\sum_{b_{i} \in D_{r}} b_{i}+\sum_{b_{i} \notin D_{r}} b_{i} \\
& \leq t N-t\left(\begin{array}{l}
r \\
2
\end{array}\right) l+(r+1-t)((r-1) l+d) \\
& \leq t N-2\left(\begin{array}{l}
r \\
2
\end{array}\right) l+(r-1)((r-1) l+d) \\
& =t N-(r-1)(l-d)<t N,
\end{aligned}
$$

again contradicting (7). We conclude that $t=1$ and suppose that $D_{r} \cap \mathscr{B}=\left\{b_{r}\right\}$, where

$$
b_{r}=N-\left(\begin{array}{l}
r \\
2
\end{array}\right) l-r z
$$

for some $z \in D$.

Our next goal is to show that all the other elements in $\mathscr{B}$ are of the form $b_{i}=i e+z, i=0, \ldots, r-1$, and here property (3) comes in handy. We begin with arguing that $t_{i}:=\left|\mathscr{B} \cap D_{i}\right|=1$ for all $i=0, \ldots, r-1$. Note that over $\mathbb{Z}$

hence from (7) and (8)

$$
\sum_{i=0}^{r-1} b_{i} \leq r((r-1) l+d)<r^{2} l<N,
$$

$$
\sum_{i=0}^{r-1} b_{i}=\left(\begin{array}{l}
r \\
2
\end{array}\right) l+r z
$$

again over $\mathbb{Z}$. Clearly, $\sum_{i=0}^{r-1} t_{i}=r$, and we will show that

$$
\sum_{i=0}^{r-1} i t_{i}=\left(\begin{array}{l}
r \\
2
\end{array}\right) \text {. }
$$

Indeed, if $\sum_{i=0}^{r-1} i t_{i} \geq\left(\begin{array}{l}r \\ 2\end{array}\right)+1$, then

$$
\begin{aligned}
l\left(\left(\begin{array}{l}
r \\
2
\end{array}\right)+1\right) & >l\left(\begin{array}{l}
r \\
2
\end{array}\right)+r d \geq\left(\begin{array}{l}
r \\
2
\end{array}\right) l+r z=\sum_{i=0}^{r-1} b_{i} \\
& =\sum_{i=0}^{r-1} \sum_{b \in \mathscr{B} \cap D_{i}} b \geq \sum_{i=0}^{r-1} \sum_{b \in \mathscr{B} \cap D_{i}} i l=\sum_{i=0}^{r-1} i t_{i} l \geq l\left(\left(\begin{array}{l}
r \\
2
\end{array}\right)+1\right),
\end{aligned}
$$

and we arrive at a contradiction. Similarly, if $\sum_{i=0}^{r-1} i t_{i} \leq\left(\begin{array}{l}r \\ 2\end{array}\right)-1$, then

$$
\begin{aligned}
\left(\begin{array}{l}
r \\
2
\end{array}\right) l & <\left(\begin{array}{l}
r \\
2
\end{array}\right) l+r z=\sum_{i=0}^{r-1} \sum_{b \in \mathscr{B} \cap D_{i}} b \leq \sum_{i=0}^{r-1} \sum_{b \in \mathscr{B} \cap D_{i}}(i l+d) \\
& =\sum_{i=0}^{r-1} t_{i}(i l+d) \leq\left(\left(\begin{array}{l}
r \\
2
\end{array}\right)-1\right) l+r d<\left(\begin{array}{l}
r \\
2
\end{array}\right) l,
\end{aligned}
$$


and (11) makes no sense, and thus (9) holds.

Finally, recalling (4), let

$$
\mathscr{B} \cap D_{i}=\left\{i l+b_{i, j}, 1 \leq j \leq t_{i}\right\}
$$

where the $b_{i, j}$ 's are $t_{i}$ distinct elements of $D$. Then over $\mathbb{Z}$

$$
\left(\begin{array}{l}
r \\
2
\end{array}\right) l+r z=\sum_{i=0}^{r-1} b_{i}=\sum_{i=0}^{r-1} \sum_{j=1}^{t_{i}}\left(i l+b_{i, j}\right)=\left(\begin{array}{l}
r \\
2
\end{array}\right) l+\sum_{i=0}^{r-1} \sum_{j=1}^{t_{i}} b_{i, j},
$$

hence

$$
\sum_{i=0}^{r-1} \sum_{j=1}^{t_{i}} b_{i, j}=r z .
$$

Now (3) implies that $b_{i, j}=z$ for all $i, j$. However, the numbers $b_{i}$ were chosen distinct, and thus, $t_{i}=1$ for all $i \leq r$. Moreover, $b_{i}=i l+z, i=0,1, \ldots, r-1$. On account of (8) and (5) the proof is complete.

Proof of Lemma 4. We closely follow [1], adding some details. Let $h$ be an integer, to be chosen later. Consider a set of integer numbers $D=\left(x_{i}\right)_{i}$ written in the form $x_{i}=\sum_{j=0}^{t} x_{i, j} h^{j}$, where $0 \leq x_{i, j}<\frac{h}{r}, i=0,1, \ldots, t$, $t=\left\lfloor\log _{h} m\right\rfloor-1$, and suppose further that for every $x_{i} \in D$

$$
\sum_{j=0}^{t} x_{i, j}^{2}=B
$$

If an $(r+1)$-tuple $x_{0}, x_{1}, \ldots, x_{r+1}$ satisfies (3), then for every $j=0,1, \ldots, t$

$$
x_{0, j}+x_{1, j}+\cdots+x_{r-1, j}=r x_{r, j} .
$$

By the convexity of the function $z \mapsto z^{2}$ this implies that

$$
x_{0, j}^{2}+x_{1, j}^{2}+\cdots+x_{r-1, j}^{2} \geq r x_{r, j}^{2},
$$

with equality if and only if $x_{0, j}=x_{1, j}=\cdots=x_{r, j}$. At the same time,

$$
\sum_{i=0}^{r-1} \sum_{j=0}^{t} x_{i, j}^{2}=r B=r \sum_{j=0}^{t} x_{r, j}^{2} .
$$

The last two relations imply that only identical $(r+1)$-tuples satisfy (12), and thus only identical $(r+1)$-tuples of elements in $D$ satisfy (3).

Clearly, $B \leq(t+1) \frac{h^{2}}{r^{2}}$, so there is a choice of $B$ such that

$$
|D| \geq \frac{h^{t+1}}{r^{t+1}(t+1) \frac{h^{2}}{r^{2}}} \geq \frac{m}{h^{3} r^{t-1}(t+1)} .
$$

Take $h=\left\lfloor e^{\sqrt{\log m \log r}}\right\rfloor$, then $(t-1) \log r<\sqrt{\log m \log r}$ and

$$
h^{3} r^{t-1}(t+1) \leq e^{5 \sqrt{\log m \log r}} .
$$

ACKNOWLEDGMENTS. Alexander Barg was partially supported by NSF grants CCF2110113 and CCF2104489. Itzhak Tamo was supported by the European Research Council (ERC grant number 852953) and by the Israel Science Foundation (ISF grant number 1030/15). 


\section{REFERENCES}

[1] N. Alon. Testing subgraphs in large graphs. In Proceedings 42nd IEEE Symposium on Foundations of Computer Science, pages 434441,2001

[2] F. A. Behrend. On sets of integers which contain no three terms in arithmetical progression. Proc. Nat. Acad. Sci. U.S.A., 32:331-332, 1946.

[3] H. Cai, Y. Miao, M. Schwartz, and X. Tang. A construction of maximally recoverable codes with order-optimal field size. arXiv:2011.13606 2020.

[4] R. Gabrys, E. Yaakobi, M. Blaum, and P. H. Siegel. Constructions of partial MDS codes over small fields. IEEE Transactions on Information Theory, 65(6):3692-3701, 2019.

[5] P. Gopalan, C. Huang, B. Jenkins, and S. Yekhanin. Explicit maximally recoverable codes with locality. IEEE Trans. Inform. Theory, 60(9):5245-5256, 2014.

[6] S. Gopi, V. Guruswami, and S. Yekhanin. Maximally recoverable LRCs: a field size lower bound and constructions for few heavy parities. IEEE Trans. Inform. Theory, 66(10):6066-6083, 2020.

[7] V. Guruswami, L. Jin, and C. Xing. Constructions of maximally recoverable local reconstruction codes via function fields. IEEE Transactions on Information Theory, 66(10):6133-6143, 2020.

[8] U. Martínez-Peñas and F. R. Kschischang. Universal and dynamic locally repairable codes with maximal recoverability via sum-rank codes. IEEE Trans. Inform. Theory, 65(12):7790-7805, 2019.

[9] A. Neri and A.-L. Horlemann-Trautmann. Random construction of partial MDS codes. Des. Codes Cryptogr., 88(4):711-725, 2020.

Department of ECE AND Institute For Systems Research, University of MARYland, College Park, MD 20742 ,

USA AND INST. FOR PROBL. Inform. TRANS., MOSCOW, RUSSIA

Email address: abarg@umd.edu

Email address: chenztan@gmail.com

Department of EE-Systems, Tel Aviv University, Tel Aviv, IsRael

Email address: zactamo@gmail.com 